\title{
Expression, localisation and potential significance of aquaporins in benign and malignant human prostate tissue
}

\author{
Johannes Bründl ${ }^{1 *}$ D, Sabine Wallinger ${ }^{1}$, Johannes Breyer ${ }^{1}$, Florian Weber $^{2}$, Matthias Evert ${ }^{2}$, \\ Nikolaos Theodoros Georgopoulos ${ }^{3}$, Bernd Rosenhammer ${ }^{1}$, Maximilian Burger ${ }^{1}$, Wolfgang Otto \\ and Peter Rubenwolf ${ }^{1,4}$
}

\begin{abstract}
Background: To study the expression pattern, localisation and potential clinical significance of aquaporin water channels (AQP) both in prostate cancer (PC) cell lines and in benign and malignant human prostate tissue.

Methods: The AQP transcript and protein expression of HPrEC, LNCaP, DU-145 and PC3 cell lines was investigated using reverse transcriptase polymerase chain reaction (RT-PCR) and immunofluorescence (IF) microscopy labelling. Immunohistochemistry (IHC) was performed to assess AQP protein expression in surgical specimens of benign prostatic hyperplasia as well as in PC. Tissue mRNA expression of AQPs was quantified by single-step reverse transcriptase quantitative polymerase chain reaction (qPCR). Relative gene expression was determined using the $40-\Delta C_{T}$ method and correlated to clinicopathological parameters.

Results: Transcripts of AQP 1, 3, 4, 7, 8, 10 and 11 were expressed in all four cell lines, while AQP 9 transcripts were not detected in malignant cell lines. IF microscopy confirmed AQP 3, 4, 5, 7 and 9 protein expression. IHC revealed highly heterogeneous AQP 3 protein expression in PC specimens, with a marked decrease in expression in tumours of increasing malignancy. Loss of AQP 9 was shown in PC specimens. mRNA expression of AQP3 was found to be negatively correlated to PSA levels ( $\rho=-0.354 ; p=0.013)$, D'Amico risk stratification $(\rho=-0.336 ; p=0.012)$, ISUP grade $(\rho=-0.321 ; p=0.017)$ and Gleason score $(\rho=-0.342 ; p=0.011)$.

Conclusions: This is the first study to systematically characterize human prostate cell lines, benign prostatic hyperplasia and PC in relation to all 13 members of the AQP family. Our results indicate the differential expression of several AQPs in benign and malignant prostate tissue. A significant correlation was observed between AQP 3 expression and tumour grade, with progressive loss in more malignant tumours. Taken together, AQPs may play a role in the progression of PC and AQP expression patterns may serve as a prognostic marker.
\end{abstract}

Keywords: Aquaporins, Human prostate, Prostate cancer, Prostate cancer cell lines

\section{Background}

Water and solute movement across the epithelia lining the male reproductive tract are essential prerequisites for seminal fluid formation and homeostasis, and are of paramount significance for the modulation of the luminal environment in which sperm cells mature and reside.

\footnotetext{
* Correspondence: johannes.bruendl@ukr.de

'Department of Urology, Caritas St Josef Medical Center, University of Regensburg, Landshuter Straße 65, 93053 Regensburg, Germany Full list of author information is available at the end of the article
}

The mechanism by which water crosses through epithelial borders had remained a matter of debate until the discovery and elucidation of the function of the aquaporin water channels (AQP) by the later Nobel laureate Peter Agre in the early 1990s. AQPs are a family of transmembrane pore-forming proteins that selectively allow water and other small, uncharged molecules such as urea, glycerol and pyrimidines to pass along hydrostatic and osmotic gradients. They play a fundamental role in numerous physiological processes, most notably in fluid absorption and secretion. To date, 13 different

(c) The Author(s). 2018 Open Access This article is distributed under the terms of the Creative Commons Attribution 4.0 International License (http://creativecommons.org/licenses/by/4.0/), which permits unrestricted use, distribution, and 
mammalian AQPs have been identified at the molecular level and localised in specific tissues [1]. Analysis of several human diseases has confirmed that AQPs are functionally involved in various pathological conditions and thus may provide promising drug targets [2]. Moreover, there is strong presumptive evidence that AQPs play a role in carcinogenesis, specifically in tumour angiogenesis and cell migration [3].

To date, the presence and significance of AQPs in the human prostate remain largely uninvestigated, with reports of the individual expression of AQP 1, 3, 5 and 9 documented in previous studies [4-8]. These findings suggest that fluid reabsorption and secretion in the prostate could be modulated by AQPs. Yet, despite investigations examining expression for individual AQPs, the human prostate has not been systematically studied in relation to all 13 members of the AQP family.

The principal aim of this study was to systematically characterize the expression pattern of all 13 AQP channels in cultured normal and malignant prostate epithelial cells, as well as freshly-isolated benign and malignant human prostate tissues. This approach allowed us to systematically study expression both at the mRNA and protein level of the whole AQP family in prostate tissue, and to correlate the pattern of expression with clinicopathological parameters. The potential biological and clinical significance of our findings are discussed.

\section{Methods}

\section{Human prostate cell lines Cell culture}

Four established human prostate cell lines, one normal human prostate epithelial cell line (HPrEC, Lifeline Cell Technology, USA), and three cancer cell lines, PC3 (Lifeline Cell Technology, USA), DU145 and LNCaP (CLS Cell Lines Service GmbH, Germany) were grown to confluency under standard culture conditions as follows: HPrEC: ProstaLife ${ }^{\mathrm{Tm}}$ Basal Medium + ProstaLife ${ }^{\mathrm{Tx}}$ LifeFactors Kit $\left(37{ }^{\circ} \mathrm{C}\right.$; $\left.5 \% \mathrm{CO}_{2}\right)$; LNCaP/DU-145/PC3: DMEM + RPMI (1:1), 5\% FBS (fetal bovine serum), $1 \%$ L-Glutamine $\left(37{ }^{\circ} \mathrm{C} ; 5 \% \mathrm{CO}_{2}\right)$.

\section{Ribonucleic acid isolation and transcript analysis (RT-PCR)} Confluent cultures of prostate cell lines were rinsed twice in phosphate-buffered saline (PBS) and harvested for the isolation of total RNA according to the manufacturer's recommendations. RNA was extracted using RNeasy Mini Kit (Qiagen) after incubation with Proteinase $\mathrm{K}$ for $10 \mathrm{~min}$. RNA quantity was assessed using a Nanodrop spectrometer (Nanodrop 2000c, Thermo Scientific). RNA from cell lines was reverse transcribed to cDNA using the iScript cDNA Synthesis Kit (Biorad) in line with the manufacturer's protocol. AQP 0-12 primers were designed using the National Centre for
Biotechnology Information (NCBI) database resources (primer sequences specified in Additional file 1: Table S1) with $ß$-actin serving as the transcript control. RT-PCR conditions were as follows: $3 \mathrm{~min}$ at $95^{\circ} \mathrm{C}$, followed by 30 cycles at $95{ }^{\circ} \mathrm{C}$ for $10 \mathrm{~s}$, annealing for $30 \mathrm{~s}$ (specific annealing temperatures specified in Additional file 1: Table S1), extension at $72{ }^{\circ} \mathrm{C}$ for $30 \mathrm{~s}$, and $8 \mathrm{~min}$ elongation at $72{ }^{\circ} \mathrm{C}$. PCR products were analysed with $2 \%$ agarose gel electrophoresis exactly as described in [9]. Reverse transcriptase (RT)-positive and RT-negative controls were included in all PCR reactions $[9,10]$.

\section{Immunofluorescence (IF) microscopy}

Cells from all four human prostate cell lines were seeded at $1 \times 10^{5}$ cells $/ \mathrm{ml}$ onto Multiwell glass slides. Cultures were fixed in a 1:1 $(\mathrm{v} / \mathrm{v})$ solution of methanol and acetone, air-dried, and incubated sequentially with the primary antibody for $16 \mathrm{~h}$ at $4{ }^{\circ} \mathrm{C}$ (see Additional file 2: Table S2) and the secondary antibody conjugated with Alexa 594 (Molecular Probes) for $30 \mathrm{~min}$, with PBS washing between steps. Hoechst 33258 (DAPI; $0.1 \mathrm{mg}$ / $\mathrm{ml}$ ) was used to stain nuclei. Secondary antibody-only negative controls and positive control cell lines known to express the respective antigen were included as specificity controls $[9,10]$. Immunolabelling was visualised by epifluorescence on a Zeiss Axio Imager Z1 microscope.

\section{Human prostate specimens Collection of specimens}

The collection of tissues had the approval of the local research ethics committee (reference number: 17-660-101) and written patient consent was also obtained. Human prostate samples were obtained from 61 patients who underwent a suprapubic adenomectomy for benign prostatic hyperplasia (BPH, $n=15)$ or a robot-assisted radical prostatectomy for biopsy-proven prostate cancer $(n=46)$ at our department in 2014. None of these patients had undergone hormonal therapy prior to surgery.

\section{Clinicopathological data}

Clinicopathological data are summarized in Table 1. All surgical specimens were assessed histopathologically by two independent uropathologists for grading and staging based on the criteria of the 2009 UICC TNM classification and ISUP 2014 Gleason grade groups and subsequently prepared for microdissection [11].

\section{Ribonucleic acid isolation}

Paraffin wax-embedded samples were de-paraffinized in xylene and microdissected samples from five serial sections $(10 \mu \mathrm{m})$ were pooled and collected in $50 \mathrm{ml}$ of lysis buffer (Qiagen). RNA was extracted using a FFPE RNA Kit (Qiagen). 
Table 1 Patient characteristics

\begin{tabular}{|c|c|}
\hline & $n(\%)$ \\
\hline \multicolumn{2}{|l|}{ Patient data } \\
\hline Age (years; range) & $66(47-84)$ \\
\hline Total number & $61(100 \%)$ \\
\hline Benign prostatic hyperplasia (BPH) & $15(24.6 \%)$ \\
\hline Low-risk PC (D'Amico) & $16(26.2 \%)$ \\
\hline Intermediate PC (D’Amico) & $16(26.2 \%)$ \\
\hline High-risk PC (D’Amico) & $14(23.0 \%)$ \\
\hline \multicolumn{2}{|l|}{ PSA } \\
\hline$<4 \mathrm{ng} / \mathrm{ml}$ & $2(3.3 \%)$ \\
\hline $4-10 \mathrm{ng} / \mathrm{ml}$ & $36(59.0 \%)$ \\
\hline $10-20 \mathrm{ng} / \mathrm{ml}$ & $12(19.7 \%)$ \\
\hline$>20 \mathrm{ng} / \mathrm{ml}$ & $5(8.2 \%)$ \\
\hline $\mathrm{n} / \mathrm{a}$ & $6(9.8 \%)$ \\
\hline \multicolumn{2}{|l|}{ ISUP (Gleason-Score) } \\
\hline $1(6)$ & $17(27.9 \%)$ \\
\hline $2(7 a)$ & $14(23.0 \%)$ \\
\hline $3(7 b)$ & $1(1.6 \%)$ \\
\hline $4(8)$ & $3(4.9 \%)$ \\
\hline $5(9-10)$ & $11(18.0 \%)$ \\
\hline No cancer & $15(24.6 \%)$ \\
\hline \multicolumn{2}{|l|}{ T-stage } \\
\hline pT2a & $7(11.5 \%)$ \\
\hline pT2b & $1(1.6 \%)$ \\
\hline pT2c & $28(45.9 \%)$ \\
\hline $\mathrm{pT} 3 / 4$ & $10(16.4 \%)$ \\
\hline No cancer & $15(24.6 \%)$ \\
\hline
\end{tabular}

Real-time quantitative polymerase chain reaction (qPCR) RNA from microdissected tissue was reverse transcribed and amplified using the iTaq Universal SYBR Green One-Step Kit (Biorad) and real-time PCR reaction was carried out on a CFX Connect Real-Time PCR Detection System (Biorad) using SYBR-Green I chemistry. Quantification was performed on MicroAmp Optical 96-Well Reaction plates. Detection of PCR products was accomplished by measuring the emitting fluorescence at the end of each reaction step. Forty amplification cycles were applied and the cycle threshold $\left(C_{T}\right)$ values of AQP 3, AQP 4, AQP 7, AQP 9 were determined along with one reference gene for each AQP. The SYBR-Green assay module includes a final melting point analysis that followed the 40 cycles of quantitative PCR. Plots from the melting point analysis were manually inspected for all RNA gene assays tested to verify that primers were specific (data not shown). PBGD (porphobilinogen desaminase) was used as housekeeping gene as previously described [12]. $\mathrm{C}_{\mathrm{T}}$ values were normalized by subtracting the $C_{T}$ value of the housekeeping gene from the $C_{T}$ value of the target gene $\left(\Delta C_{T}\right)$. RNA results were then reported as $40-\Delta C_{T}$ values to ensure that the normalized gene expression obtained by the test was proportional to the corresponding mRNA expression levels [13, 14].

\section{Immunohistochemistry}

Surgical samples were fixed in $10 \%$ formalin, dehydrated, and embedded in paraffin wax. Dewaxed 4- $\mu \mathrm{m}$ tissue sections were subjected to antigen retrieval by boiling for $10 \mathrm{~min}$ in tris-ethylenediaminetetraacetic acid (Tris-EDTA, pH 9 for AQP 3) or citric acid ( $\mathrm{pH} 6$ for AQP $4,5,7$, and 9), before labelling with pre-titrated primary antibodies (see Additional file 2: Table S2) for $16 \mathrm{~h}$ at $4^{\circ}$ C. Secondary antibody-only controls and positive control tissues known to express the respective antigen were included as specificity controls $[9,10]$.

\section{Statistical analysis}

Statistical analyses were performed using SPSS version 23. The Spearman's rank correlation coefficient $\rho$ was used as a measure of the strength and direction of the relationship between variables. Levels of mRNA-expression were stratified by quartiles.

\section{Results}

AQP expression by human prostate cell cultures in vitro AQP gene expression was investigated by RT-PCR and immunofluorescence microscopy. In vitro, normal human prostate epithelial cells (HPrEC) as well as malignant cell lines (LNCaP, DU-145 and PC3) showed expression of AQP 1, 3, 4, 7, 8, 10 and 11 (see Fig. 1). By contrast, transcripts for AQP 0,2 , and 12 were not detected (not shown). AQP 5 mRNA transcripts were detected in the DU-145 and PC3 cancer cell lines, but not in LNCaP or HPrEC cells. Transcripts of AQP 6 were present in all cell lines except in LNCaP cells. AQP 9 transcripts were found in HPrEC, but not in malignant cell lines.

Using immunofluorescence microscopy, AQP 3, 4 and 7 were detected in normal cells as well as the three PC cell lines, whereas AQP 5 was only expressed in DU-145 and PC3 cancer cell lines, in line with the RT-PCR analysis results. Expression of AQP 9 was found in normal prostate cells (HPrEC), but was absent in all three cancer cell lines (LNCaP, DU-145, PC3), also in accordance with the transcript findings. Representative results of these experiments are shown in Fig. 2. The remaining AQPs could not be assessed for protein expression due to the lack of suitable commercially available antibodies.

\section{AQP expression by native human prostate tissue}

Having detected AQP expression at the mRNA and protein level in normal and malignant cell lines in vitro, the 


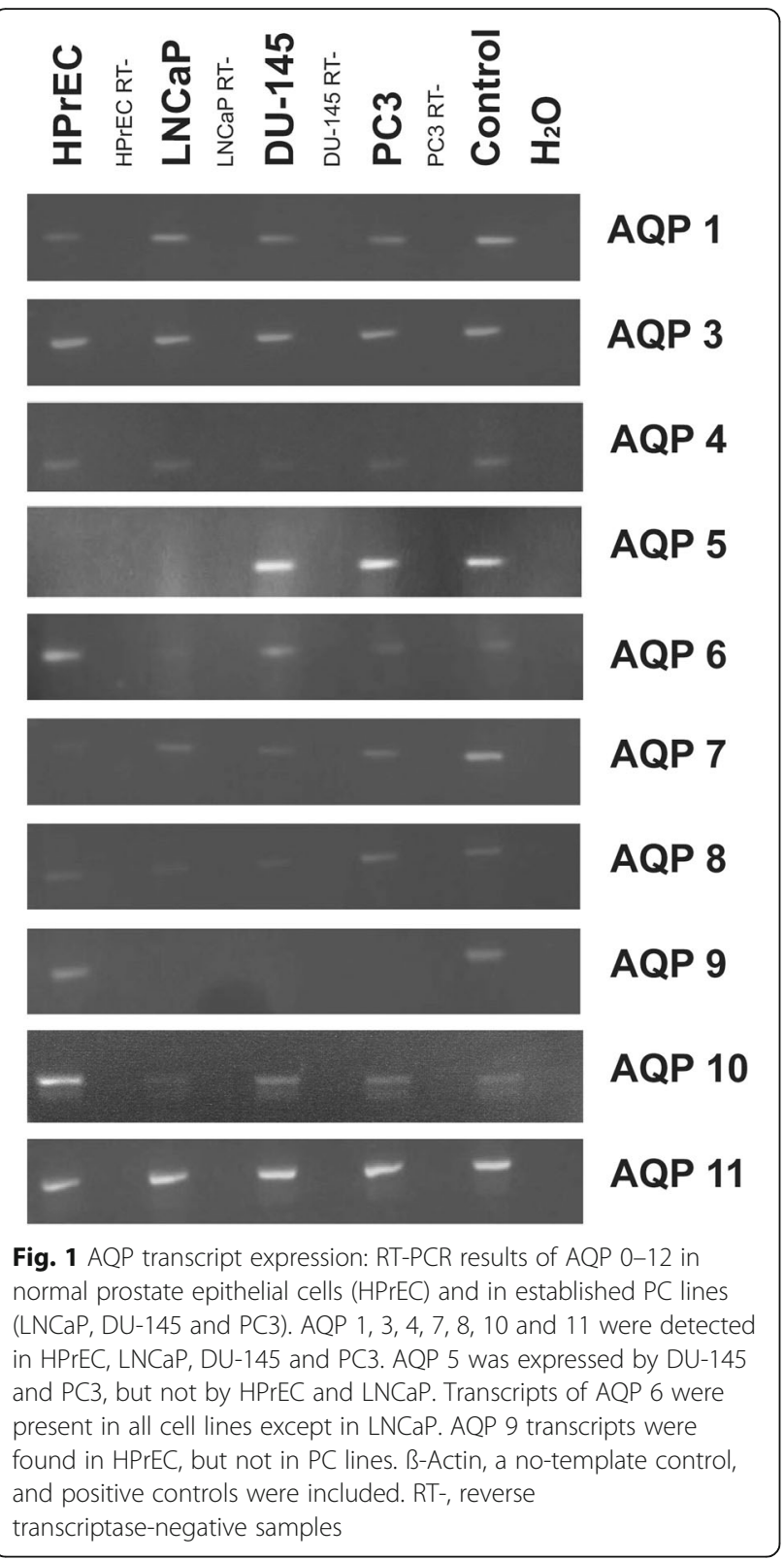

level of expression and cellular localisation of AQP protein in native, non-malignant prostate tissue was examined by immunohistochemistry. AQP 3 was strongly expressed throughout the epithelium of all specimens with benign prostatic hyperplasia $(\mathrm{BPH})$. Labelling was most intense in the basal layer, less intense in the intermediate layer and weak in the luminal cells. At higher magnification, a distinct localisation pattern was apparent, with intense labelling at the intercellular borders in the basal and suprabasal compartments, as shown in Fig. 3. No immunoreactivity was seen in the submucosa, smooth muscle or endothelium. AQP 4 was not detected in any $\mathrm{BPH}$ specimen. AQP 5, AQP 7 and AQP 9 were found to be present in the epithelium of all benign tissues, although the expression pattern was less unequivocal compared with AQP 3 (Fig. 3).

In contrast to our observations in non-malignant $(\mathrm{BPH})$ tissue, a highly heterogeneous AQP 3 protein expression was observed in prostate cancer specimens, with marked decrease in intensity and expression in tumours of increasing malignancy. Whereas intense expression with distinct labelling of the cell borders was present in the epithelium of low-risk tumours, less intense expression of $\mathrm{AQP} 3$ with alternating AQP-positive and AQP-negative tumour cells was found in intermediate-risk tumours. Extensive AQP-negative compartments and abrupt transition from strongly labelled to unlabelled tumour cells were evident in tumours of high malignancy. Complete loss of expression of AQP 3 was found in one third of high-risk cancers. AQP 4 was expressed in half of the tumours tested, and expression was independent of the ISUP/Gleason grade groups. Likewise, heterogenous expression of AQP 5 and AQP 7 was found in all tumours independent of malignancy grades. Finally, AQP 9 was not detected in malignant prostatic tissue. Representative results of these studies are shown in Fig. 4.

\section{Correlation of mRNA expression of AQP 3, AQP 4, AQP 7 and AQP 9 with clinicopathological parameters}

AQP transcript expression of human prostate specimen was quantified by qPCR. The non-parametric Spearman's rank correlation indicated a negative, statistically significant correlation between mRNA expression of AQP 3 and PSA levels $(\rho=-0.354 ; p=0.013)$, D'Amico risk stratification $(\rho=-0.336 ; p=0.012)$, ISUP grade $(\rho=-0.321 ; p=0.017)$ and Gleason score $(\rho=-0.342$; $p=0.011$ ). Expression of AQP 4 revealed a statistically significant positive correlation with the presence of tumour ( $\rho=0.493 ; p=0.001$ ), D'Amico risk stratification $(\rho=0.436 ; p=0.003)$, ISUP grade $(\rho=0.434 ; p=0.003)$ and Gleason score $(\rho=0.436 ; p=0.003)$ but not with PSA $(\rho=0.094 ; p=0.577)$ and tumour stage $(\rho=0.270$; $p=0.080)$. By contrast, there was no correlation between AQP 7 mRNA expression and any clinicopathological parameter investigated. Expression of AQP 9 transcripts was found to be negatively correlated with PSA $(\rho=-0.366 ; p=0.010)$. Please refer to Fig. 5 .

\section{Discussion}

The expression and function of AQPs has been investigated in the majority of human tissues [9]. Previous findings have suggested that fluid reabsorption and secretion in numerous organs are modulated by AQPs. However, human prostate tissue has not yet been systematically analysed in relation to AQP channel expression. The objective of the present study was to systematically investigate the expression and localisation of AQPs not only in human prostate (normal and malignant) cell lines in vitro, but 


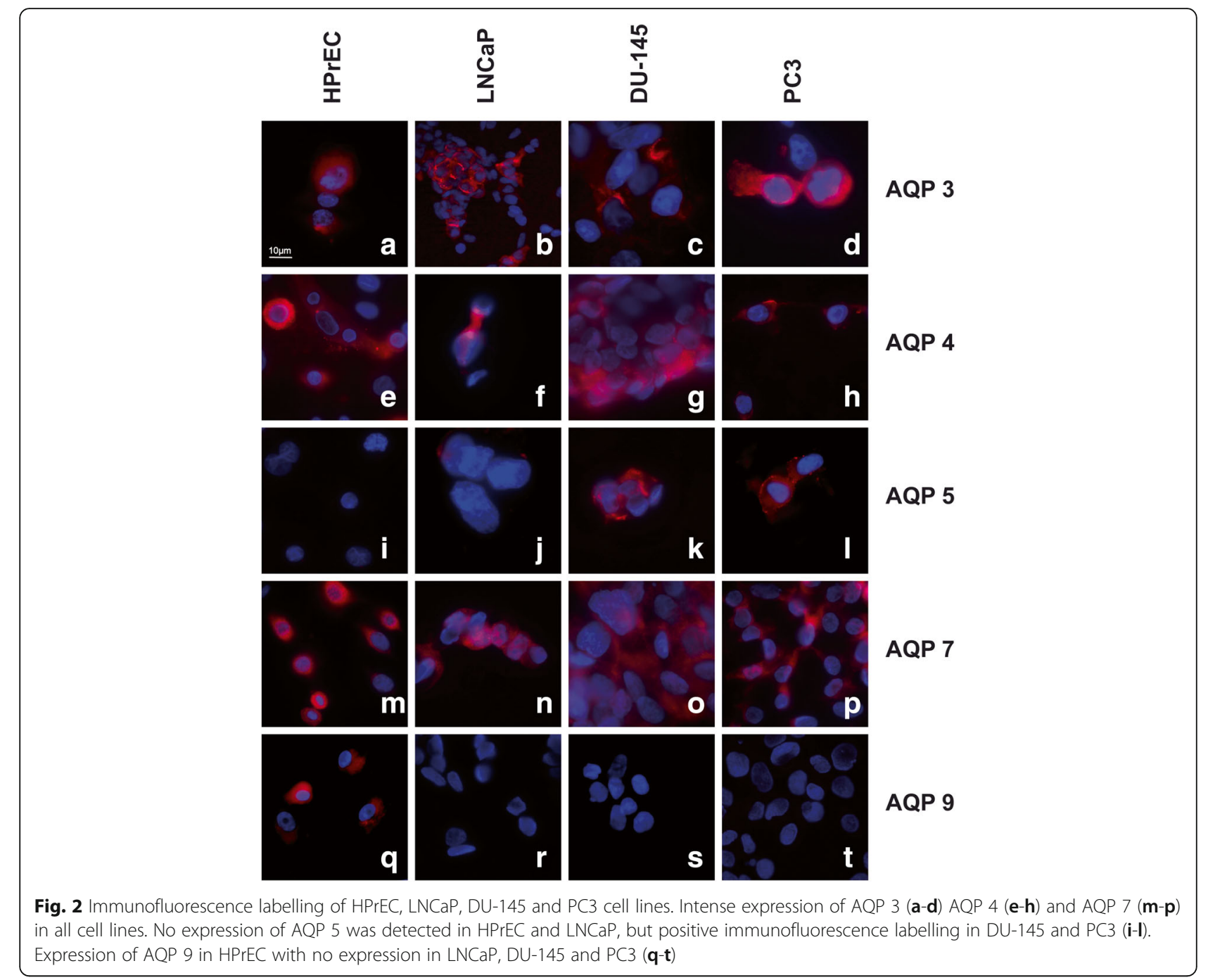

also to investigate AQP expression in surgical specimens of $\mathrm{BPH}$ and $\mathrm{PC}$ of various malignancy grades.

Our study is the first to characterize human prostate tissue in relation to all 13 members of the AQP family. Transcripts for AQP 1, 3, 4, 7, 8, 10 and 11 were consistently detected in all four cell lines, while there was differential expression of AQP 5, 6 and 9. AQP 0, 2 and 12 were not detected in our study. Our mRNA transcript findings were corroborated at the protein level by immunofluorescence microscopy, where we detected AQP protein expression for those AQP family members where commercially available antibodies were able to be used. Our mRNA and protein findings in vitro are collectively in agreement with previous studies, which showed expression of AQP 1, 3, 5 in the human prostate at both the transcript and protein levels $[7,15]$. Having characterized AQP expression in normal and malignant human prostate cell lines, we then investigated AQP expression in human $\mathrm{BPH}$ and $\mathrm{PC}$ specimens of various tumour grades via $\mathrm{qPCR}$ and immunohistochemistry.

The expression of AQPs by human prostate tissue and recent reports on their possible role in carcinogenesis in epithelial tissues raise interesting questions about the potential functional (biological) significance of AQPs in the development and progression of PC. We contend that it is particularly important to investigate whether the pattern (and localisation) of AQP expression could be of prognostic and/or of therapeutic value. Our results provide strong presumptive evidence that there is a correlation between the loss of AQP 3 expression and increased PC tumour grade. To our knowledge, this is the first report of progressive loss of AQP 3 expression in high-grade PC. Previous investigations into the significance of AQPs in non-urological tumours have almost invariably shown overexpression of AQP 3 and it has been hypothesized that AQP 3 may be a promising drug 

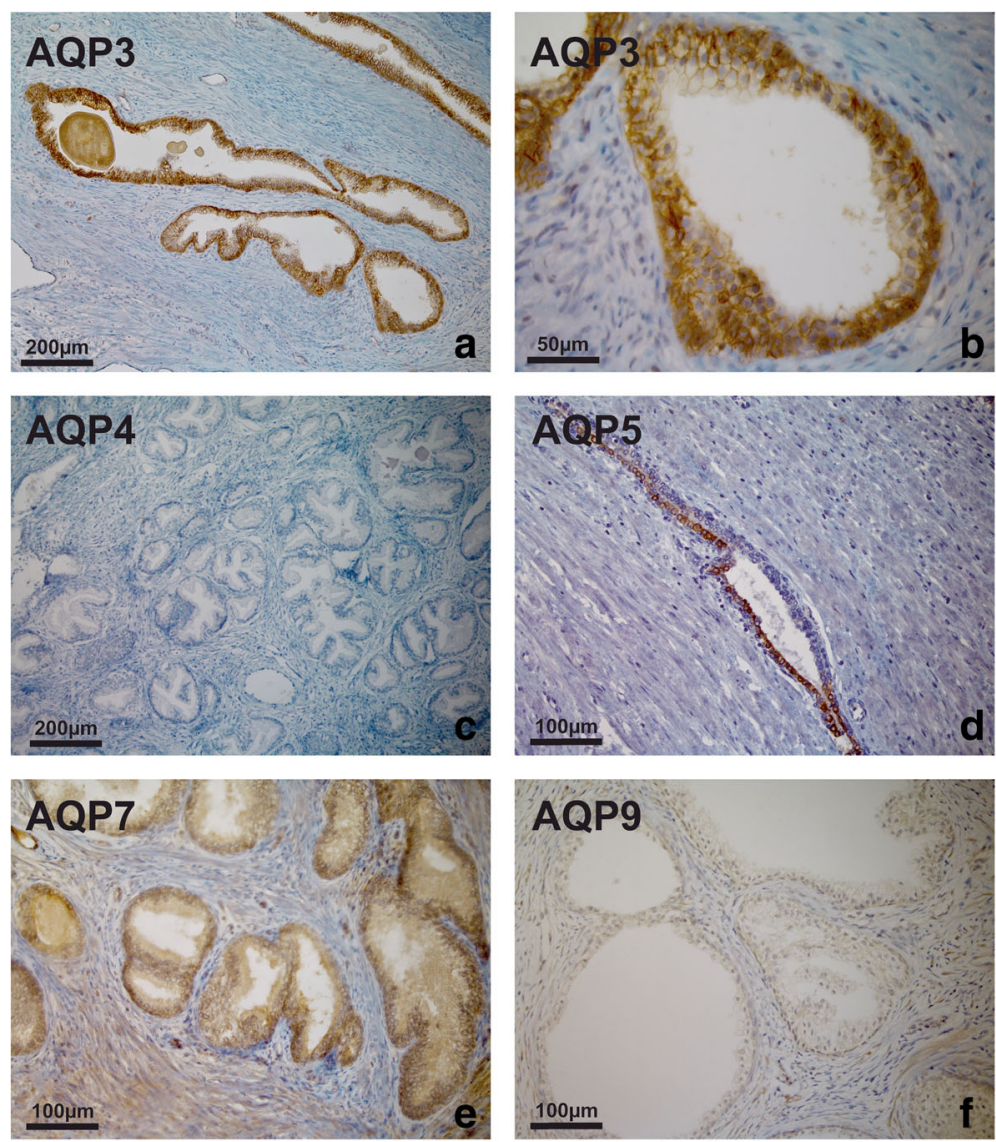

Fig. 3 AQP immunoperoxidase labelling of non-malignant prostatic tissues (BPH). Intense expression of AQP 3 throughout the epithelium from a patient with $\mathrm{BPH}$. Labelling was most intense in the basal layer, less intense in the intermediate layer and weak in the luminal cells (a). At higher magnification, a distinct localisation pattern was apparent, with intense labelling of intercellular borders in the basal and suprabasal compartments (b). No immunoreactivity was seen in the submucosa, smooth muscle or endothelium. No expression of AQP 4 was detected (c). Heterogeneous expression of AQP 5 (d), diffuse expression of AQP 7 (e) and weak expression of AQP 9 (f) was observed

target in the treatment of various epithelial tumours [16-19]. The contrasting expression pattern of AQP 3 with intense labelling of AQP 3 in the case of BPH and well-differentiated $\mathrm{PC}$, but non-homogeneous expression or loss of AQP 3 in the case of high-risk tumours, is noteworthy and may reflect tumour heterogeneity. Although this does not completely corroborate with cell line data, it is well known that in vitro cell lines do not necessarily reflect what happens in vivo. PC is well known for its tumour heterogeneity, which is reflected by diverse morphological manifestations and various molecular alterations associated with different tumour phenotypes [20].

With regard to clinicopathological parameters, downregulation of AQP3 was associated with higher preoperative PSA values, higher risk according to the D'Amico classification and a higher Gleason-/ISUP-grade. Interestingly, in support of our prostate cancer findings here, we previously reported a similar observation in urothelial carcinoma, with studies suggesting that the loss of AQP 3 may play a role in bladder cancer progression. We showed a significant correlation between AQP 3 protein expression and tumour stage and grade, with AQP 3 expression being reduced or lost in urothelial carcinomas of higher grade and stage [10,21]. Furthermore, loss of AQP 3 expression was associated with worse progress ion-free and cancer-specific survival in patients with muscle-invasive bladder cancer [22].

The pro-tumorigenic effect of AQP 3 loss has also been reported for non-urological tumour entities. For instance, knockdown of AQP 3 expression resulted in increased migration and proliferation in gastric adenocarcinoma cell lines [16]. By contrast, overexpression of AQP 3 has been demonstrated for most other tumour entities, such as for squamous cell carcinomas [17]. Although establishing a functional role for AQP 3 expression in BPH and PC was beyond the scope of this study, our findings suggest that progressive loss of AQP 3 expression may be associated with worse clinical PC outcomes. 

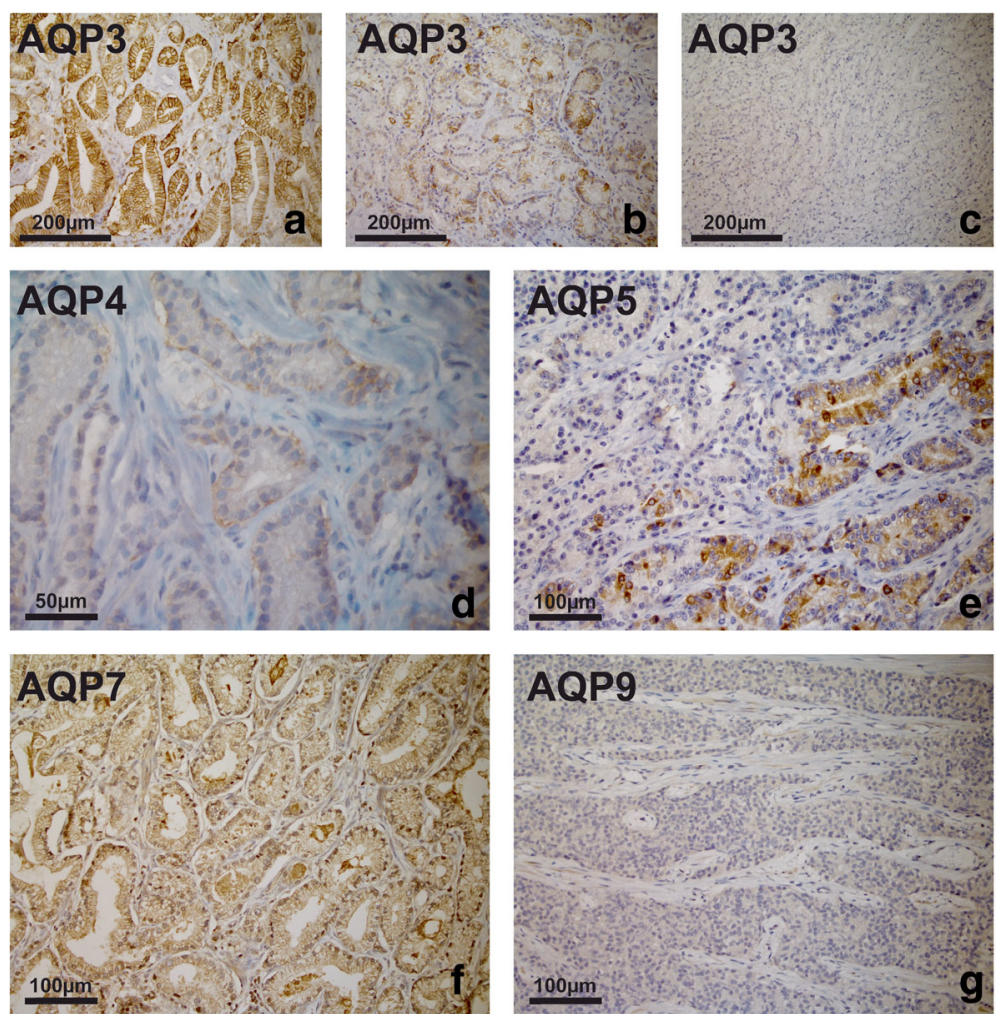

Fig. 4 AQP immunoperoxidase labelling of prostate cancer specimens. Intense expression with distinct labelling at the cell borders in a sample of a low-risk tumour (a), less intense expression of AQP 3 with alternating AQP-positive and AQP-negative tumour cells found in an intermediate-risk tumour (b). Complete loss of AQP 3 expression in a high-risk tumour (c). Weak expression of AQP 4 in an intermediate-risk tumour (d). Heterogeneous expression of AQP 5 (e) and expression of AQP 7 (f) in PC. AQP 9 was not detected (g)

When studying AQP 4 expression, we found expression in half of carcinomas but not in any BPH specimens in immunohistochemistry. Moreover, a significant positive correlation was observed between mRNA expression in qPCR and D'Amico risk classification, Gleason- and ISUP-grading. Differential expression of AQP 4 in benign and cancerous tissue and its potential biological and clinical roles need to be elucidated in further studies.
AQP 5 and 7 were detected both in $\mathrm{BPH}$ and in PC specimens of all grades. However, there was no correlation of AQP 5 and 7 mRNA expression with any clinicopathological parameter in our cohort. These findings are similar to the observations made in a recent study by Park et al. [15]. Pust et al. previously reported highly variable AQP 5 expression in PC with both negative and intense expression of AQP 5 being linked to unfavourable outcomes

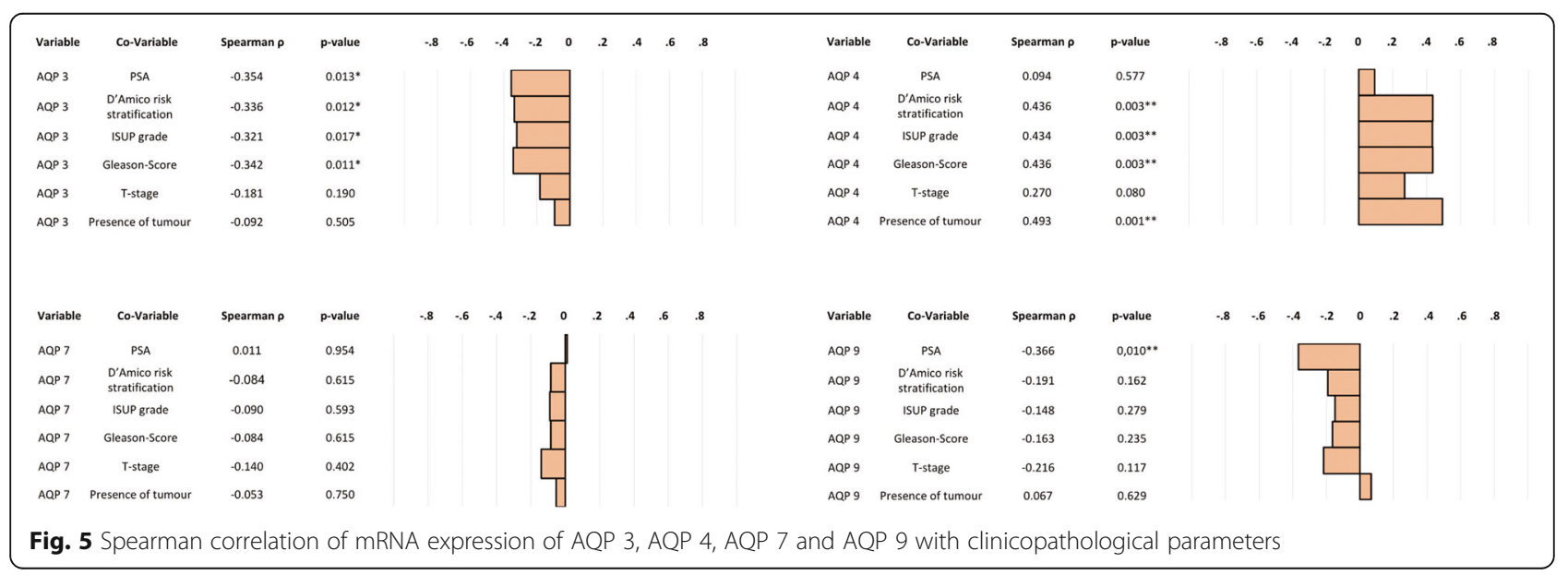


[23]. In contrast to our findings, Li et al. demonstrated that AQP 5 expression was upregulated and associated with advanced stage, circulating tumour cells and inferior survival rates in PC [24]. These discrepancies and a wealth of somewhat contradictory results are interesting, and indicate that further studies are required to scrutinize the significance of AQP 5 expression in PC.

AQP 9 has previously been shown to be ubiquitously expressed along the male reproductive tract [25]. In concordance with this, we found expression of AQP 9 in normal prostatic cells in vitro and in BPH in vivo, whereas loss of expression was consistently demonstrated in cancer cell lines and in PC specimens. The demonstration of an inverse correlation of AQP 9 mRNA levels in qPCR and PSA supports this. Our study is the first to report the consistent loss of AQP 9 expression in PC, the biological significance of which still remains to be established. In hepatocellular cancer, for instance, decreased expression of AQP 9 resulted in increased resistance to apoptosis [26]. In rat prostates, AQP 9 expression has been shown to be regulated by androgens [8]. Similarly, Jiang et al. and Tian et al. suggested that expression of AQP 1,3-8, 10-12 in PC is modified by androgens, at least in rats [27, 28]. However, there are little published data on its role in the human prostate. Hence, the potential role of AQP 9 in the carcinogenesis of PC needs to be addressed in future studies.

Despite the clear differential expression of AQP demonstrated in our study (particularly AQP 3), we do accept that our observational investigation was conducted on a relatively limited number of $\mathrm{BPH}$ and $\mathrm{PC}$ specimens. As such, despite their significance, our findings would certainly be strengthened by a bigger cohort of clinical specimens. One definite weakness of our study, however, is the lack of healthy prostate tissue serving as a control. Hence, we can only speculate on the relationship between AQP expression and function in normal human prostate tissue. Nevertheless, we did observe clear differential expression of AQP and a correlation with increasing malignancy in PC. While such an approach goes beyond the scope of the present study, future studies must certainly seek to prove a functional role for AQP 3 in PC progression. This would involve mechanistic studies and silencing with the RNAi-mediated knockdown of AQP 3 to assess biological endpoints relevant to tumour progression (proliferation, migration/invasion, and/or resistance to apoptotic stimuli) in the PC cell lines studied here. In addition, pre-clinical PDX models from patients with castration resistant PC might also be helpful. This would provide mechanistic insight into how changes in AQP expression may regulate tumour biology. We are currently addressing such questions, and are carrying out further studies that include sufficient patient numbers and long-term survival data to elucidate the potential clinical significance of AQP expression and its role in PC.

\section{Conclusions}

This is the first study to demonstrate that several AQPs are expressed in human prostate cell lines, BPH and PC. Our results indicate differential expression of several AQPs in benign and malignant prostate tissue. A significant correlation was observed between AQP 3 protein expression and tumour grade, with a progressive loss of AQP 3 expression in more malignant tumours. However, it has yet to be determined whether AQPs play defined biological role(s) in the initiation and/or progression of $\mathrm{PC}$ and, more specifically, whether this could be of prognostic or therapeutic value.

\section{Additional files}

Additional file 1: Table S1. RT-PCR oligonucleotide primers. Complete list of RT-PCR oligonucleotide primers used throughout this study. (DOCX $19 \mathrm{~kb}$ )

Additional file 2: Table S2. Antibodies used for immunofluorescence (IF) and immunohistochemistry (IHC) studies. Complete list of antibodies used for immunofluorescence (IF) and immunohistochemistry (IHC) throughout this study. (DOCX $17 \mathrm{~kb}$ )

\section{Abbreviations}

AQP: Aquaporin; BPH: Benign prostatic hyperplasia; CDNA: Complementary desoxyribonucleic acid; DMEM: Dulbecco modified Eagle's minimal essential medium; FBS: Fetal bovine serum; IF: Immunofluorescence; IHC: Immunhistochemistry; ISUP: International Society of Urological Pathology; mRNA: Messenger ribonucleic acid; PBGD: Porphobilinogen desaminase; PBS: Phosphate-buffered saline; PC: Prostate cancer; PDX: Patient derived xenograft; qPCR: Quantitative polymerase chain reaction; RNA: Ribonucleic acid; RPMl: Roswell Park Memorial Institute medium; RTPCR: Reverse-transcriptase polymerase chain reaction; TNM: TNM Classification of Malignant Tumours; Tris-EDTA: Tris-

ethylenediaminetetraacetic acid; UICC: Union internationale contre le cancer

\section{Acknowledgments}

The authors would like to thank Stefanie Götz and Nina Mierswa for excellent technical and Ralph Wirtz for statistical support.

\section{Availability of data and materials}

All data generated or analysed during this study are included in this published article (and its supplementary information files).

\section{Authors' contributions}

All authors have read and approved the manuscript. Study concept and design: JB1 (Johannes Bründl), SW, ME, MB, WO, PR. Acquisition of data: JB1, JB2 (Johannes Breyer), BR. Analysis and interpretation of data: SW, FW, PR. Drafting of the manuscript: JB1, SW, NG, PR. Critical revision of the manuscript for important intellectual content: JB2, ME, NG, BR, MB, WO, PR. Statistical analysis: JB1, JB2. Administrative, technical, or material support: SW, WO. Supervision: MB, ME, PR.

\section{Ethics approval and consent to participate}

The study was performed following approval of the local research ethics committee at the University of Regensburg and written patient consent (reference number: 17-660-101; Ethics committee, University of Regensburg, 93,040 Regensburg, Germany).

Consent for publication

Not applicable.

Competing interests

All authors have declared that they have no competing interests. 


\section{Publisher's Note}

Springer Nature remains neutral with regard to jurisdictional claims in published maps and institutional affiliations.

\section{Author details}

'Department of Urology, Caritas St Josef Medical Center, University of Regensburg, Landshuter Straße 65, 93053 Regensburg, Germany. ${ }^{2}$ Institute of Pathology, University of Regensburg, Regensburg, Germany. ${ }^{3}$ Department of Biological Sciences, School of Applied Sciences, University of Huddersfield, Huddersfield, UK. ${ }^{4}$ Department of Urology, Frankfurt University Medical Center, Frankfurt, Germany.

Received: 22 May 2018 Accepted: 27 August 2018

Published online: 03 September 2018

\section{References}

1. Magni F, Sarto C, Ticozzi D, Soldi M, Bosso N, Mocarelli P, Kienle MG. Proteomic knowledge of human aquaporins. Proteomics. 2006;6(20):5637.

2. Jeyaseelan K, Sepramaniam S, Armugam A, Wintour EM. Aquaporins: a promising target for drug development. Expert Opin Ther Targets. 2006; 10(6):889.

3. Saadoun S, Papadopoulos MC, Hara-Chikuma M, Verkman AS. Impairment of angiogenesis and cell migration by targeted aquaporin-1 gene disruption. Nature. 2005:434(7034):786

4. Hwang I, Jung SI, Hwang EC, Song SH, Lee HS, Kim SO, Kang TW, Kwon D, Park K. Expression and localization of aquaporins in benign prostate hyperplasia and prostate cancer. Chonnam medical journal. 2012;48(3):174

5. Mobasheri A, Airley R, Hewitt SM, Marples D. Heterogeneous expression of the aquaporin 1 (AQP1) water channel in tumors of the prostate, breast, ovary, colon and lung: a study using high density multiple human tumor tissue microarrays. Int J Oncol. 2005;26(5):1149,

6. Mobasheri A, Marples D, Young IS, Floyd RV, Moskaluk CA, Frigeri A. Distribution of the AQP4 water channel in normal human tissues: protein and tissue microarrays reveal expression in several new anatomical locations, including the prostate gland and seminal vesicles. Channels. 2007;1 (1):29.

7. Wang J, Tanji N, Kikugawa T, Shudou M, Song X, Yokoyama M. Expression of aquaporin 3 in the human prostate. Int J Urol. 2007;14(12):1088.

8. Wang J, Tanji N, Sasaki T, Kikugawa T, Song X, Yokoyama M. Androgens upregulate aquaporin 9 expression in the prostate. Int J Urol. 2008; 15(10):936.

9. Rubenwolf PC, Georgopoulos NT, Clements LA, Feather S, Holland P, Thomas DF, Southgate J. Expression and localisation of aquaporin water channels in human urothelium in situ and in vitro. Eur Urol. 2009;56(6):1013.

10. Rubenwolf PC, Otto W, Denzinger S, Hofstadter F, Wieland W, Georgopoulos NT. Expression of aquaporin water channels in human urothelial carcinoma: correlation of AQP3 expression with tumour grade and stage. World J Urol. 2014:32(4):991.

11. Mottet N, Bellmunt J, Bolla M, Briers E, Cumberbatch MG, De Santis M, Fossati N, Gross T, Henry AM, Joniau S, et al. EAU-ESTRO-SIOG quidelines on prostate Cancer. Part 1: screening, diagnosis, and local treatment with curative intent. Eur Urol. 2017;71(4):618.

12. Boormans JL, Hermans KG, Made AC, van Leenders GJ, Wildhagen MF, Collette L, Schroder FH, Trapman J, Verhagen PC. Expression of the androgen-regulated fusion gene TMPRSS2-ERG does not predict response to endocrine treatment in hormone-naive, node-positive prostate cancer. Eur Urol. 2010;57(5):830.

13. Breyer J, Wirtz RM, Otto W, Erben P, Worst TS, Stoehr R, Eckstein M, Denzinger S, Burger M, Hartmann A. High PDL1 mRNA expression predicts better survival of stage pT1 non-muscle-invasive bladder cancer (NMIBC) patients. Cancer Immunol Immunother. 2018;67(3):403.

14. Breyer J, Wirtz RM, Otto W, Laible M, Schlombs K, Erben P, Kriegmair MC Stoehr R, Eidt S, Denzinger S, et al. Predictive value of molecular subtyping in NMIBC by RT-GPCR of ERBB2, ESR1, PGR and MKI67 from formalin fixed TUR biopsies. Oncotarget. 2017:8(40):67684

15. Park JY, Yoon G. Overexpression of Aquaporin-1 is a prognostic factor for biochemical recurrence in prostate adenocarcinoma. Pathol Oncol Res. 2017;23(1):189

16. Huang Y, Zhu Z, Sun M, Wang J, Guo R, Shen L, Wu W. Critical role of aquaporin-3 in the human epidermal growth factor-induced migration and proliferation in the human gastric adenocarcinoma cells. Cancer Biol Ther. 2010;9(12):1000.

17. Kusayama M, Wada K, Nagata M, Ishimoto S, Takahashi H, Yoneda M, Nakajima A, Okura M, Kogo M, Kamisaki Y. Critical role of aquaporin 3 on growth of human esophageal and oral squamous cell carcinoma. Cancer Sci. 2011;102(6):1128

18. Niu D, Kondo T, Nakazawa T, Yamane T, Mochizuki K, Kawasaki T, Matsuzaki T, Takata K, Katoh R. Expression of aquaporin3 in human neoplastic tissues. Histopathology. 2012;61(4):543.

19. Verkman AS, Hara-Chikuma M, Papadopoulos MC. Aquaporins--new players in cancer biology. J Mol Med (Berl). 2008;86(5):523.

20. Tolkach Y, Kristiansen $\mathrm{G}$. The heterogeneity of prostate Cancer: a practical approach. Pathobiology. 2018;85(1-2):108.

21. Rubenwolf PC, Denzinger S, Otto W. Aquaporin 3 protein expression in transitional cell carcinoma: a potential marker with regard to tumour progression and prognosis? Eur Urol. 2012;61(3):627.

22. Rubenwolf $\mathrm{P}$, Thomas $\mathrm{C}$, Denzinger S, Hartmann A, Burger M, Georgopoulos $N T$, Otto W. Loss of AQP3 protein expression is associated with worse progression-free and cancer-specific survival in patients with muscleinvasive bladder cancer. World J Urol. 2015;33(12):1959.

23. Pust A, Kylies D, Hube-Magg C, Kluth M, Minner S, Koop C, Grob T, Graefen M, Salomon G, Tsourlakis MC, et al. Aquaporin 5 expression is frequent in prostate cancer and shows a dichotomous correlation with tumor phenotype and PSA recurrence. Hum Pathol. 2016; https://doi.org/10.1016/j. humpath.2015.09.026.

24. Li J, Wang Z, Chong T, Chen H, Li H, Li G, Zhai X, Li Y. Over-expression of a poor prognostic marker in prostate cancer: AQP5 promotes cells growth and local invasion. World J Surg Oncol. 2014; https://doi.org/10.1186/14777819-12-284.

25. Pastor-Soler N, Bagnis C, Sabolic I, Tyszkowski R, McKee M, Van Hoek A, Breton S, Brown D. Aquaporin 9 expression along the male reproductive tract. Biol Reprod. 2001;65(2):384.

26. Jablonski EM, Mattocks MA, Sokolov E, Koniaris LG, Hughes FM Jr, Fausto N, Pierce RH, McKillop IH. Decreased aquaporin expression leads to increased resistance to apoptosis in hepatocellular carcinoma. Cancer Lett. 2007; 250(1):36

27. Jiang J, Tian JC, Xia JY, Zhu YS, Jiang R. Expressions of aquaporins decrease in the prostate and seminal vesicles of castrated rats. Zhonghua Nan Ke Xue. 2015;21(4):300.

28. Tian JC, Xia JY, Jiang J, Jiang R, He YZ, Lin H. Effect of androgen deprivation on the expression of aquaporins in rat prostate and seminal vesicles. Andrologia. 2016;48(3):268.
Ready to submit your research? Choose BMC and benefit from:
- fast, convenient online submission
- thorough peer review by experienced researchers in your field
- rapid publication on acceptance
- support for research data, including large and complex data types
- gold Open Access which fosters wider collaboration and increased citations
- maximum visibility for your research: over $100 \mathrm{M}$ website views per year
At $\mathrm{BMC}$, research is always in progress.
Learn more biomedcentral.com/submission 